\title{
Synovial fluid of patients with rheumatoid arthritis induces $\alpha$-smooth muscle actin in human adipose tissue-derived mesenchymal stem cells through a TGF- $\beta 1$-dependent mechanism
}

\author{
Hae Young Song ${ }^{1}$, Min Young Kim ${ }^{1}$, \\ Kyung Hye Kim ${ }^{1}$, II Hwan Lee ${ }^{1}$, Sang Hun Shin ${ }^{1}$, \\ Jung Sub Lee ${ }^{2}$ and Jae Ho Kim ${ }^{1,3}$ \\ ${ }^{1}$ Medical Research Center for Ischemic Tissue Regeneration \\ Department of Physiology \\ Medical Research Institute \\ ${ }^{2}$ Department of Orthopaedic Surgery \\ School of Medicine, Pusan National University \\ Yangsan 626-870, Korea \\ ${ }^{3}$ Corresponding author: Tel, 82-51-510-8073; \\ Fax, 82-51-510-8076; E-mail, jhkimst@pusan.ac.kr \\ DOI 10.3858/emm.2010.42.8.057 \\ Accepted 12 July 2010 \\ Available Online 13 July 2010
}

\begin{abstract}
Abbreviations: hASCs, human adipose tissue-derived mesenchymal stem cells; LPA, lysophosphatidic acid; MSCs, mesenchymal stem cells; RA, Rheumatoid arthritis; RA-SF, synovial fluids from patients with RA; SF, synovial fluid; shRNA, short hairpin RNA; siRNA, small interfering RNA; SPC, sphingosylphosphorylcholine; $\alpha$-SMA, $\alpha$-smooth muscle actin
\end{abstract}

\begin{abstract}
Rheumatoid arthritis (RA) is a chronic, inflammatory autoimmune disorder that causes the immune system to attack the joints. Transforming growth factor- $\beta 1$ (TGF- $\beta 1$ ) is a secreted protein that promotes differentiation of synovial fibroblasts to $\alpha$-smooth muscle actin ( $\alpha$-SMA)-positive myofibroblasts to repair the damaged joints. Synovial fluid from patients with RA (RA-SF) induced expression of $\alpha$-SMA in human adipose tissue-derived mesenchymal stem cells (hASCs). RA-SF-induced $\alpha$-SMA expression was abrogated by immunodepletion of TGF- $\beta 1$ from RA-SF with anti-TGF- $\beta 1$ antibody. Furthermore, pretreatment of hASCs with the TGF- $\beta$ type I receptor inhibitor SB431542 or lentiviral small hairpin RNA-mediated silencing of TGF- $\beta$ type I receptor expression in hASCs blocked RA-SF-induced $\alpha$-SMA expression. Small interfering RNA-mediated silencing of Smad2 or adenoviral overexpression of Smad7 (an inhibitory Smad
\end{abstract}

isoform) completely inhibited RA-SF-stimulated $\alpha$-SMA expression. These results suggest that TGF- $\beta 1$ plays a pivotal role in RA-SF-induced differentiation of hASCs to $\alpha$-SMA-positive cells.

Keywords: mesenchymal stem cells; rheumatoid arthritis; synovial fluid; transforming growth factor $\beta 1$; $\alpha$-smooth muscle actin

\section{Introduction}

Rheumatoid arthritis (RA) is a chronic inflammatory disease characterized by destruction of articular cartilage and adjacent bone tissues (Feldmann et al., 1996) and disordered synovial microenvironment, including infiltration of inflammatory cells, hyperplasia of stromal cells, and tissue scarring (Buckley, 2003). Synovial fluid (SF), which nourishes articular cartilage and lubricates articular joint surfaces (Ghosh and Guidolin, 2002), contains various growth factors and cytokines (Szekanecz et al., 1998). The pathological events of RA are mediated by a complex interplay of pro-inflammatory cytokines and mediators produced in the joint tissues or synovium of patients with RA (Scrivo et al., 2007).

Transforming growth factor- $\beta 1$ (TGF- $\beta 1$ ), a member of the TGF- $\beta$ super family of cytokines, is involved in diverse cellular responses, such as proliferation, differentiation, apoptosis, and production of extracellular matrix (Wahl, 2007). TGF- $\beta$ is important for the induction of fibrosis associated with RA (Pohlers et al., 2009). Alpha-smooth muscle actin ( $\alpha$-SMA)-positive myofibroblasts reportedly play a key role in the fibrosis and pathogenesis of RA (Kasperkovitz et al., 2005) and TGF- $\beta 1$ is involved in the differentiation of synovial fibroblasts to $\alpha$-SMA-positive cells (Mattey et al., 1997; Steenvoorden et al., 2006). TGF- $\beta$ ligand binding induces the heterodimeric association of TGF- $\beta$ type I and II serine/threonine receptor kinases (Shi and Massague, 2003; ten Dijke and Hill, 2004). Activated TGF- $\beta$ type I receptor recruits and phosphorylates Smad2 and Smad3, which in turn associate with the protein Smad4 (Shi and 
A

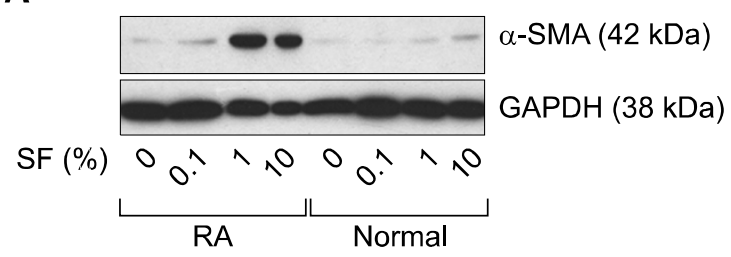

C

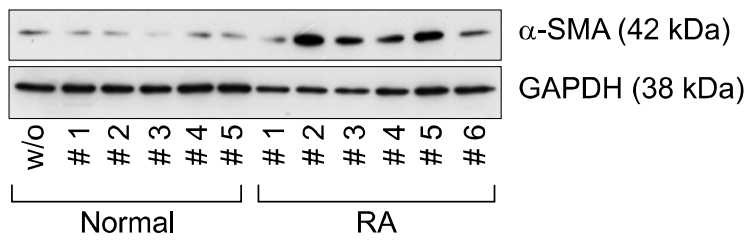

B

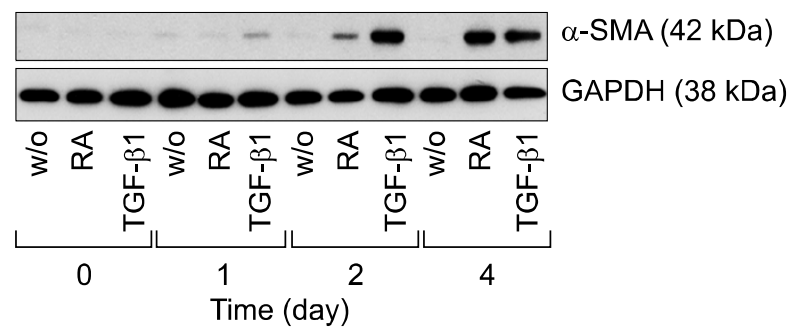

D

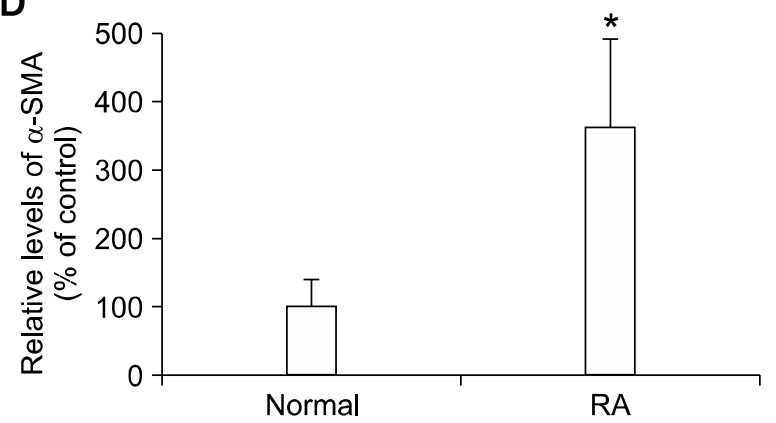

Figure 1. Effects of RA-SF on the expression of $\alpha$-SMA in hASCs. (A) Serum-starved hASCs were treated with indicated concentrations of SF with RA patients or normal patients for 4 days. (B) Serum-starved hASCs were treated with vehicle, $1 \%$ RA-SF or $0.2 \mathrm{ng} / \mathrm{ml}$ TGF- $\beta 1$ for the defined times. (C) Serum-starved hASCs were treated with vehicle (w/o), $1 \%$ SF of five normal donors, or $1 \%$ SF of six RA patients for 4 days. (D) The densities of $\alpha$-SMA were quantified from three independent experiments, and the expression levels of $\alpha$-SMA were normalized to total GAPDH levels in the samples. The data are presented as a percentage of control. ${ }^{*}, P<0.05$.

Massague, 2003; ten Dijke and Hill, 2004). Complexed Smads translocate to the nucleus, where they regulate transcription of TGF- $\beta$-responsive genes, whereas Smad7 inhibits intracellular signaling by the TGF- $\beta$ superfamily proteins, mainly by interacting with activated type I receptors for the TGF- $\beta$ superfamily proteins (Attisano and Wrana, 2000; de Caestecker, 2004).

Mesenchymal stem cells or multipotent stromal cells (MSCs) possess self-renewal capacity, long-term viability, and differentiation potential toward diverse cell types, such as adipogenic, osteogenic, chondrogenic, and myogenic lineages (Prockop, 1997; Pittenger et al., 1999; Short et al., 2003; Barry and Murphy, 2004), suggesting a potential application of MSCs for regenerative medicine. MSCs have been suggested to play a key role in rheumatology, based on their differentiation potential to cartilage/bone and their capacity to modulate host immune responses, angiogenesis, and fibrosis (Djouad et al., 2009). Originally discovered in the bone marrow, MSCs have now been identified in various joint tissues, including joint fat, periosteum, synovial membrane, SF, and cartilage (Bouffi et al., 2009; Djouad et al., 2009). MSCs have been reported to reside in the SF of patients with arthritis (Jones et al., 2004) and their migration to the synovium has been demonstrated in a collagen-induced arthritis animal model (Marinova-Mutafchieva et al., 2002). We recently reported that synovial fluids from patients with RA (RA-SF) stimulates migration of human bone marrow-derived MSCs (hBMSCs) through a lysophosphatidic acid (LPA)-mediated mechanism in vitro (Song et al., 2010). Furthermore, human adipose tissue-derived mesenchymal stem cells (hASCs) can differentiate to $\alpha$-SMA-positive cells in response to treatment with LPA or TGF- $\beta 1$ (Jeon et al., 2008). These results raise the possibility that differentiation of MSCs to $\alpha$-SMA-positive cells can be regulated by the RA-associated synovial microenvironment. To address this, we examined the effects of RA-SF on the expression of $\alpha$-SMA in hASCs as a model system for tissue-resident MSCs. Herein, we report on the identification of TGF- $\beta 1$ as a key factor of RA-SF that induces $\alpha$-SMA expression.

\section{Results}

\section{RA-SF induces expression of $\alpha$-SMA in hASCs}

To explore whether RA-SF can induce differentiation of hASCs to $\alpha$-SMA-positive cells, hASCs were treated with different concentrations of SF from RA patients or normal donors. As shown in 

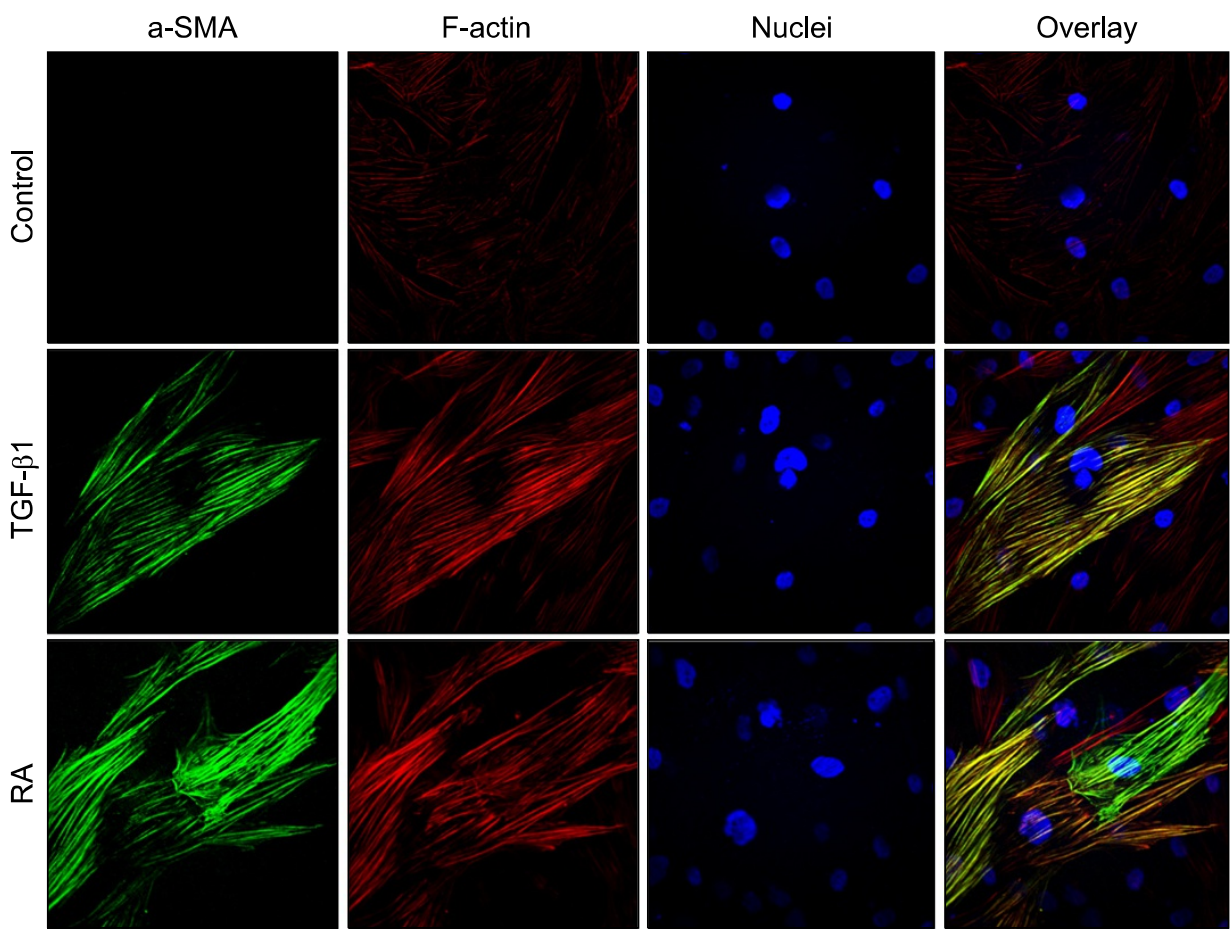

Figure 2. Effects of RA-SF on the formation of actin stress fibers and the localization of $\alpha$-SMA in hASCs. Serum-starved hASCs were treated with vehicle, $1 \%$ RA-SF, or $0.2 \mathrm{ng} / \mathrm{ml}$ TGF- $\beta 1$ for 4 days, and immunofluorescence staining was performed. $\alpha$-SMA was stained with anti- $\alpha$-SMA antibody and probed with Alexa Fluor 488-conjugated anti-mouse secondary antibody. F-actin was detected with Alexa Fluor 568 phalloidin and the double stained images were analyzed by confocal microscope $(400 \times$ magnification). The merged images of $\alpha$-SMA (green) and F-actin (red) are shown. Representatives of three independent experiments are shown.

Figure 1A, RA-SF induced $\alpha$-SMA expression in hASCs with a maximal stimulation at $1 \%$ concentration. However, SF from normal donors had no significant impact on $\alpha-S M A$ expression. Because TGF- $\beta 1$ is known to stimulate expression of $\alpha$-SMA in hASCs (Jeon et al., 2006), we compared the effects of RA-SF and TGF- $\beta 1$ on $\alpha$-SMA expression. $\alpha$-SMA expression was apparent on day 2 after treatment of the cells with RA-SF and was maximally induced on day 4 as potent as TGF- $\beta 1$-induced $\alpha$-SMA expression (Figure 1B). To evaluate whether RA-SF specifically increased $\alpha$-SMA expression, we next compared the effects of SF from different RA patients or normal donors. As shown in Figures. 1C and 1D, RA-SF exhibited more potent stimulatory effects on $\alpha$-SMA expression in hASCs than SF from normal donors, suggesting that RA-SF stimulates expression of $\alpha$-SMA in hASCs.

We next examined the effect of RA on intracellular distribution of $\alpha$-SMA and actin filaments by double staining for $\alpha-S M A$ and actin stress filaments. As shown in Figure 2, treatment of hASCs with RA-SF or TGF- $\beta 1$ for 4 days increased the expression level of $\alpha$-SMA, which was localized in actin filaments. These results support the idea that RA-SF induces $\alpha$-SMA expression and formation of intracellular actin filaments in hASCs.
A

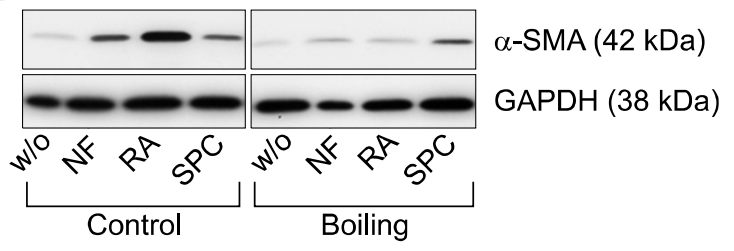

B

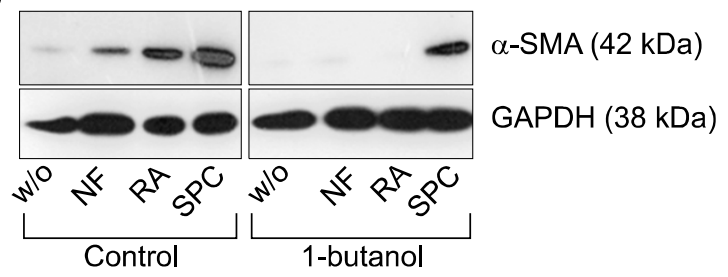

Figure 3. Role of protein components in RA-SF-induced $\alpha$-SMA expression. (A) To denature protein components, control synovial fluid (NF), RA-SF, or SPC were boiled at $95^{\circ} \mathrm{C}$ for 5 min. Serum-starved hASCs were exposed to untreated control or boiled samples $(1 \% \mathrm{NF}, 1 \% \mathrm{RA}-\mathrm{SF}$, and $5 \mu \mathrm{M} \mathrm{SPC}$ ) for 4 days. (B) Lipid components were extracted from NF, RA-SF or SPC with 1-butanol. Serum-starved hASCs were exposed to untreated controls or 1 -butanol fractions ( $1 \% \mathrm{NF}, 1 \% \mathrm{RA}-\mathrm{SF}$, and $5 \mu \mathrm{M}$ SPC) for 4 days. The expression levels of $\alpha$-SMA and GAPDH were analyzed by Western blot. Representatives of three independent experiments are shown. 




Figure 4. RA-SF induces expression of $\alpha$-SMA through LPA receptor-independent pathway. Serum-starved hASCs were pretreated with vehicles, $5 \mu \mathrm{M}$ Ki16425 for $15 \mathrm{~min}$. The cells were then exposed to vehicle, $1 \% \mathrm{NF}, 1 \%$ RA-SF, $0.2 \mathrm{ng} / \mathrm{ml}$ TGF- $\beta 1$, or $5 \mu \mathrm{M}$ LPA for 4 days, and the expression levels of $\alpha$-SMA and GAPDH were determined by Western blot. Representative of three independent experiments is shown.

\section{Protein components are responsible for RA-SF-induced $\alpha$-SMA expression}

To explore whether protein factors could be responsible for the RA-SF-induced expression of $\alpha-S M A, R A-S F$ and normal SF were heated to $95^{\circ} \mathrm{C}$ for 5 min to denature protein factors. As shown in Figure $3 \mathrm{~A}$, the stimulatory effects of RA-SF or normal SF on the $\alpha$-SMA expression of hASCs were abrogated by heating. We have reported that the lysophospholipid sphingosylphosphorylcholine (SPC) induces $\alpha$-SMA expression in hASCs (Jeon et al., 2006). In contrast, SPC-induced $\alpha$-SMA expression was not affected by heating of SPC. These results support the suggestion that protein factors are likely to be involved in the $\alpha$-SMA expression induced by RA-SF or normal SF. To support the involvement of protein factors in the RA-SF-induced $\alpha$-SMA expression, we next examined the effect of lipid fractions extracted from RA-SF with 1-butanol, on $\alpha$-SMA expression. As shown in Figure 3B, $\alpha$-SMA expression by RA-SF and normal SF was not recapitulated by the 1-butanol fractions, whereas the stimulatory effect of SPC was maintained in 1-butanol fraction. These results support the suggestion that protein factors are responsible for the RA-SF-induced $\alpha$-SMA expression.
A

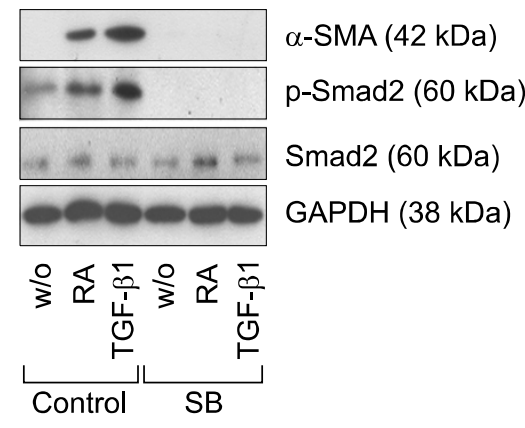

B

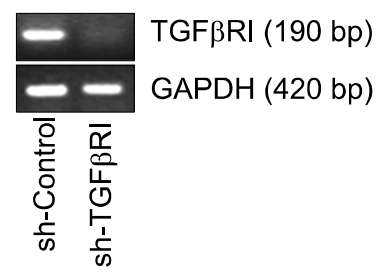

C

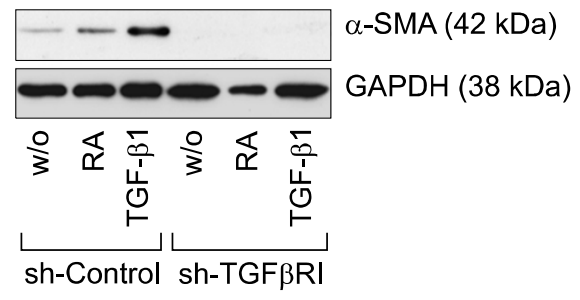

D

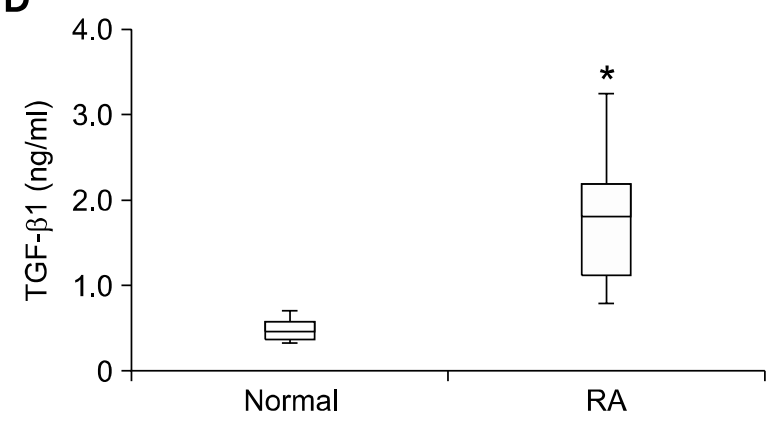

E



Figure 5. Role of TGF- $\beta 1$-dependent pathway in the RA-SF-induced $\alpha$-SMA expression. (A) Serum-starved hASCs were treated with $1 \%$ RA-SF or 0.2 $\mathrm{ng} / \mathrm{ml}$ TGF- $\beta 1$ for 4 days in the absence or in the presence of $10 \mu \mathrm{M}$ SB-431542. (B) Serum-starved hASCs were infected with control or lentiviral TGF- $\beta$ type I receptor-specific shRNA. The mRNA levels of TGF- $\beta$ type I receptor (TGF $\beta R 1)$ were determined by semi-quantitative RT-PCR. (C) Lentiviral infected cells were then exposed to vehicle, $1 \%$ RA-SF or $0.2 \mathrm{ng} / \mathrm{ml} \mathrm{TGF- \beta 1}$. (D) SF of normal donors or RA patients was subjected to ELISA for determination of the concenturations of TGF- $\beta 1$. In the statistical analysis of TGF- $\beta 1$ concentrations, box and whisker plots represent median values, $25-75 \%$ range, and $10-90 \%$ range. ${ }^{*}, P<0.05$ vs. normal. (E) $1 \%$ RA-SF or $0.2 \mathrm{ng} / \mathrm{ml}$ TGF- $\beta 1$ were incubated with $0.2 \mu \mathrm{g} / \mathrm{ml}$ anti-TGF- $\beta 1$ or control antibodies for $1 \mathrm{~h}$, and supernatants were collected after precipitation of the immune complexes for depletion of TGF- $\beta 1$. hASCs were treat with the TGF- $\beta 1$-depleted supernatants for 4 days. The expression levels of $\alpha$-SMA and GAPDH were determined by Western blot. Representatives of three independent experiments are shown. 
A

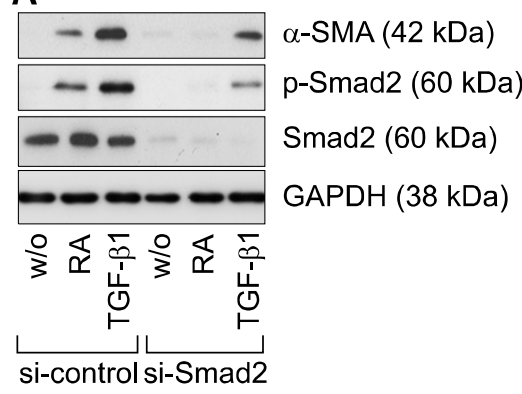

B

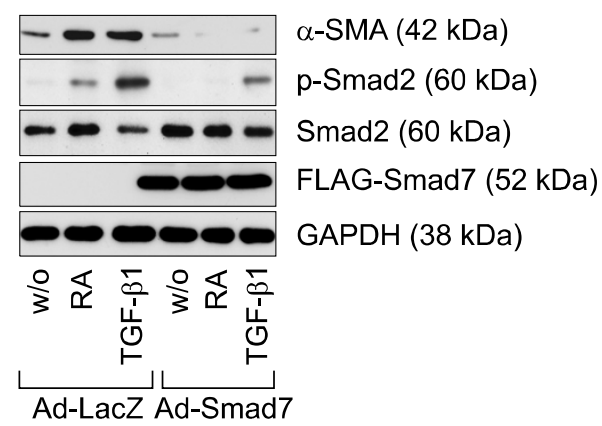

Figure 6. Role of Smad2 in the RA-SF-induced expression of $\alpha$-SMA. (A) Serum-starved hASCs were transfected with control si-RNA (Control) or Smad2 si-RNA (si- Smad2), and then exposed with 1\% RA-SF or $0.2 \mathrm{ng} / \mathrm{ml}$ TGF- $\beta 1$ for 4 days. (B) hASCs were infected with adenoviruses carrying $\beta$-galactosidase (Ad-LacZ) or FLAG-tagged Smad7 (Ad-Smad7), and then treat with vehicles or $1 \%$ RA-SF and $0.2 \mathrm{ng} / \mathrm{ml} \mathrm{TGF- \beta 1} \mathrm{for} 4$ days. The expression levels of $\alpha$-SMA, Smad2, FLAG-tagged Smad7, and GAPDH were determined by Western blotting with anti- $\alpha-S M A$, anti-Smad2, anti-FLAG and anti-GAPDH antibodies. Phosphorylation of Smad2 was determined by Western blotting with anti-p-Smad2. Representatives of three independent experiments are shown.

\section{LPA is not involved in RA-SF-induced expression of $\alpha$-SMA in hASCs}

LPA is responsible for RA-SF-induced migration of hBMSCs (Song et al., 2010). Furthermore, LPA treatment stimulates $\alpha$-SMA expression in hASCs (Jeon et al., 2008). To explore whether LPA could be involved in the RA-SF-induced $\alpha$-SMA expression, we examined the effect of the LPA receptor antagonist Ki16425 on $\alpha$-SMA expression induced by RA-SF or LPA. Ki16425 completely inhibited LPA-induced $\alpha$-SMA expression (Figure 4). On the other hand, $\alpha$-SMA expression stimulated by RA-SF or TGF- $\beta 1$ was not affected by Ki16425 treatment. These results support the notion that LPA is not responsible for RA-SF-induced expression of $\alpha$-SMA in hASCs.

\section{TGF- $\beta 1$ is involved in the RA-SF-stimulated $\alpha$-SMA expression in hASCs}

TGF- $\beta 1$ stimulates $\alpha$-SMA expression in hASCs (Jeon et al., 2006, 2008). To explore the involvement of TGF- $\beta$-dependent mechanism in the RA-SF-stimulated $\alpha$-SMA expression, we tested the effect of the TGF- $\beta$ type I receptor kinase inhibitor SB431542. As shown in Figure 5A, pretreatment of hASCs with SB431542 completely abrogated $\alpha$-SMA expression which was induced not only by TGF- $\beta 1$ but also by RA-SF. Furthermore, both RA-SF and TGF- $\beta 1$ elicited phosphorylation of Smad2 through a SB431542-sensitive mechanism. To confirm the involvement of TGF- $\beta$ type I receptor in the RA-SF-induced $\alpha$-SMA expression, we depleted endogenous TGF- $\beta$ type I receptor using lentiviral infection of TGF- $\beta$ type I receptor-specific shRNA (Figure 5B). Silencing of
TGF- $\beta$ type I receptor expression completely blocked the RA- or the TGF- $\beta 1$-induced $\alpha$-SMA expression (Figure $5 \mathrm{C}$ ). To elucidate the involvement of TGF- $\beta 1$ in the RA-SF stimulation of $\alpha$-SMA expression, we next determined the concentrations of TGF- $\beta 1$ in the SF derived from patients with RA and normal donors using ELISA. As shown in Figure 5D, TGF- $\beta 1$ levels in SF with RA were significantly increased compared with those of normal controls. Furthermore, TGF- $\beta 1$ - and RA-SF-induced $\alpha$-SMA expression were abrogated by immunodepletion of TGF- $\beta 1$ with anti-TGF- $\beta 1$ neutralizing antibody (Figure $5 \mathrm{E}$ ). These results support the suggestion that RA-SF induces $\alpha$-SMA expression in hASCs through TGF- $\beta 1-T G F-\beta$ receptor-dependent mechanism.

\section{Role of Smad2 in RA-SF-stimulated $\alpha$-SMA expression in hASCs}

Because Smad2 plays a key role in TGF- $\beta 1$-induced signaling pathways (Shi and Massague, 2003; ten Dijke and Hill, 2004), we examined the involvement of Smad2 activation in RA-SF-induced $\alpha$-SMA expression using siRNA-mediated depletion of Smad2. As shown in Figure $6 A$, depletion of endogenous Smad2 markedly attenuated $\alpha$-SMA expression and Smad2 phosphorylation which were induced by RA-SF or TGF- $\beta 1$. Adenoviral overexpression of Smad7, an inhibitory Smad isoform, blocked $\alpha$-SMA expression and Smad2 phosphorylation stimulated by RA-SF or TGF- $\beta 1$ (Figure 6B), suggesting that Smad2-dependent pathway is involved in the RA-SF induction of $\alpha$-SMA expression in hASCs. 


\section{Discussion}

It is conceivable that MSCs are useful for therapy of RA due to their immunoregulatory characteristics, differentiation potential, and paracrine function (Djouad et al., 2009; Kastrinaki and Papadaki, 2009). In the present study, we demonstrate that RA-SF can stimulate $\alpha$-SMA expression in MSCs through a TGF- $\beta 1$-dependent mechanism. The concentration of TGF- $\beta 1$ was significantly augmented in RA-SF than normal SF, and immunodepletion of endogenous TGF- $\beta 1$ from RA-SF using TGF- $\beta 1$ neutralizing antibody abrogated the RA-SF-stimulated $\alpha$-SMA expression in human adipose tissue-derived MSCs, implying that TGF- $\beta 1$ in RA-SF directly stimulates $\alpha$-SMA expression. Furthermore, pharmacological inhibition or shRNA-mediated depletion of TGF- $\beta$ type I receptor blocked RA-SF-stimulated $\alpha$-SMA expression. In addition, siRNA-mediated silencing of Smad2 or adenoviral overexpression of Smad7 suppressed RA-SF-induced $\alpha$-SMA expression by ablation of TGF- $\beta$ receptor-Smad2 signaling pathway. TGF- $\beta 1$ reportedly plays a pivotal role in fibrosis and pathogenesis associated with RA (Pohlers et al., 2009). Treatment with TGF- $\beta 1$ induces differentiation of human synovial fibroblasts to $\alpha$-SMA-positive myofibroblasts (Mattey et al., 1997), which play a key role in RA-associated synovial inflammation (Kasperkovitz et al., 2005). Synovial fibroblasts from patients with RA exhibited constitutive activation of the TGF- $\beta$ signaling pathway due to abundant expression of TGF- $\beta 1$ and TGF- $\beta$ type I receptor (Pohlers et al., 2007). Moreover, treatment with not only TGF- $\beta 1$ but also RA-SF stimulates $\alpha$-SMA expression in synovial fibroblasts (Steenvoorden et al., 2006). Because MSCs have been shown to reside in RA-SF (Jones et al., 2004), these results support the notion that TGF- $\beta 1$ plays a pivotal role in the RA-SF-induced differentiation of MSCs to $\alpha$-SMA-positive cells.

We recently reported that LPA is responsible for the RA-SF-induced migration of hBMSCs (Song et al., 2010); Pretreatment of cells with the LPA receptor antagonist Ki16425 or silencing of LPA1 receptor expression abrogated RA-SF-stimulated cell migration. RA-SF induced migration of not only hBMSCs but also hASCs through LPA-dependent mechanism (data not shown). Our present findings suggest that LPA is not involved in RA-SF-induced $\alpha$-SMA expression. Firstly, Ki16425 treatment had no significant impact on the RA-SF-induced $\alpha$-SMA expression. Secondly, heat denaturation and lipid extraction experiments demonstrated that protein components, but not lipid factors, were involved in the RA-SF-stimulated $\alpha$-SMA expression. Therefore, it is likely that LPA and TGF- $\beta 1$ mediate RA-SF-induced cell migration and $\alpha$-SMA expression, respectively. RA-SF-induced $\alpha$-SMA expression was potently stimulated by treatment with $1 \%$ RA-SF (Figure 1A), whereas RA-SF-induced cell migration was maximally stimulated at $10 \%$ concentration (Song et al., 2010). The concentrations of LPA in RA-SF are estimated to be $0.98 \pm 0.12$ $\mu \mathrm{M}$ (Song et al., 2010), therefore, the concentration of LPA in 1\% RA-SF can be calculated to be $9.8 \pm$ $0.12 \mathrm{nM}$. Because LPA treatment elicited $\alpha$-SMA expression at $2 \mu \mathrm{M}$ (Jeon et al., 2008), these results suggest that LPA concentration in $1 \%$ RA-SF is not sufficient to stimulate $\mu$-SMA expression in hASCs.

$\alpha$-SMA-positive myofibroblasts play pivotal roles in a variety of physiology and pathophysiology, including wound healing, cancer, and tissue fibrosis (Gabbiani, 2003). The present study supports the view that increased levels of TGF- $\beta 1$ in the synovial microenvironment of RA patients may locally affect the differentiation of tissue-resident MSCs within joint tissues to myofibroblast-like cells. $\alpha$-SMA is a marker for not only myofibroblasts but also smooth muscle cells, and both myofibroblasts and smooth muscle cells share similar phenotypic characteristics (De Wever et al., 2008). The importance of TGF- $\beta 1$ in RA involves an association with certain vascularization patterns in the synovial membrane (Salvador et al., 2006). While RA primarily affects joints, increasing body of evidence demonstrates that RA is associated with the development of various cardiovascular diseases including atherosclerosis, vasculitis lesions, and coronary artery disease (Tanasescu et al., 2009). The inflammatory hypothesis of cardiovascular diseases in RA implies that mediators originating from inflamed synovial tissue may have systemic vascular responses by modulating differentiation status of MSCs. More research is needed to clarify the pathophysiological significance of RA-SF-stimulated differentiation of MSCs to myofibroblast-like cells and the involvement of TGF- $\beta 1$ in the differentiation of MSCs in vivo.

\section{Methods}

\section{Materials}

$\alpha$-Minimum essential medium, phosphate-buffered saline, trypsin, fetal bovine serum, Moloney Murine Leukemia Virus (M-MLV) reverse transcriptase, and Lipofectamine plus $^{\mathrm{TM}}$ reagent were purchased from Invitrogen (Carlsbad, CA). Human recombinant TGF- $\beta 1$, anti-TGF- $\beta 1$ neutralizing antibody, and enzyme-linked immunosorbent assay (ELISA) kits for TGF- $\beta 1$ were purchased from R\&D 
Systems (Minneapolis, MN). 1-Oleoyl-sn-glycero-3- phosphate (1-oleoyl-LPA), fatty acid-free bovine serum albumin, Ki16425, SB431542, and anti- $\alpha$-SMA mouse monoclonal antibody were purchased from Sigma-Aldrich (St. Louis, MO). Anti-phospho-Smad2 (Ser465/467) and anti-Smad2 antibodies were purchased from Cell Signaling Technology (Beverly, MA). Alexa Fluor 488 goat anti-mouse antibody and Alexa Fluor 568 phalloidin were from Molecular Probes (Eugene, OR).

\section{Collection of SF}

SF was obtained with the patient's consent, as approved by the Institution Review Board of Busan National University Hospital. SF was obtained from patients with RA during therapeutic arthrocentesis as previously described (Song et al., 2010). SF from normal donors was obtained postmortem from organ donors without joint diseases. SF was transferred to heparin-treated tubes, transported immediately to the laboratory, and centrifuged at 3,000 $\times g$ for $10 \mathrm{~min}$ at $4^{\circ} \mathrm{C}$ to remove possible inflammatory cells and blood cells. Aliquots of the supernatants were used immediately or stored at $-80^{\circ} \mathrm{C}$ for future analysis. SF aliquots were either used immediately or subjected to one freeze-thaw cycle.

\section{Extraction of lipid components and denaturation of protein components of SF}

To extract lipid components, aliquots $(200 \mu \mathrm{l})$ of SF were each treated with 1 volume of 1-butanol. After vigorous shaking and centrifugation (5 $\mathrm{min}$ at $15,000 \mathrm{rpm}$ ), the upper 1-butanol phase was collected and evaporated under nitrogen. The resultant lipids were solubilized in 200 $\mu \mathrm{l}$ of $1 \mathrm{mg} / \mathrm{ml}$ fatty acid-free bovine serum albumin. To denature proteins in SF, an aliquot $(200 \mu \mathrm{l})$ of SF was heated at $100^{\circ} \mathrm{C}$ for $5 \mathrm{~min}$, centrifuged at $15,000 \mathrm{rpm}$ for 5 min to remove denatured proteins, and the supernatants were collected.

\section{Cell culture}

After informed consent, adipose tissues were obtained from patients undergoing elective abdominoplasty. For isolation of hASCs, adipose tissues were washed at least three times with sterile PBS and treated with an equal volume of $0.1 \%$ collagenase for $30 \mathrm{~min}$ at $37^{\circ} \mathrm{C}$ with intermittent shaking. The floating adipocytes were separated from the stromal-vascular fraction by centrifugation at $300 \times g$ for $5 \mathrm{~min}$. The cell pellet was filtered through a $100-\mathrm{mm}$ nylon mesh to remove cellular debris and incubated overnight at $37^{\circ} \mathrm{C}$ in an atmosphere of $5 \%$ $\mathrm{CO}_{2}$ in $\alpha$-minimum essential medium containing $10 \%$ fetal bovine serum, $100 \mathrm{units} / \mathrm{ml}$ of penicillin, and $100 \mu \mathrm{g} / \mathrm{ml}$ streptomycin. Following incubation, the plates were washed extensively with PBS to remove residual nonadherent red blood cells. When the monolayer of adherent cells reached confluence, the primary hASCs were subcultured at a concentration of $2 \times 10^{3} \mathrm{cells} / \mathrm{cm}^{2}$. The primary hASCs were cultured for 4-5 days until they reached confluence and were defined as passage 0 . The passage number of hASCs used in these experiments was 3-10. The hASCs were positive for CD29, CD44, CD90, and CD105, all of which have been reported to be mesenchymal stem cells marker proteins. However, these cells did not express c-kit, CD34, or CD14, which are known as hematopoietic markers (data not shown).

\section{Immunocytochemistry and microscopy}

Immunostaining and confocal microscopy were used to determine the subcellular distribution and organization of proteins. Cells were fixed in PBS containing 4\% paraformaldehyde for $15 \mathrm{~min}$, permeabilized with PBS containing $0.2 \%$ Triton $\mathrm{X}-100$ for $10 \mathrm{~min}$, and blocked with PBS containing $2 \%$ bovine serum albumin. For immunostaining, specimens were incubated with anti- $\alpha-S M A$ antibody for $2 \mathrm{~h}$ and Alexa Fluor 488-conjugated anti-mouse secondary antibody for $1 \mathrm{~h}$. To stain F-actin filaments, the specimen was incubated with Alexa Fluor 543-conjugated phalloidin for $30 \mathrm{~min}$ followed by confocal microscopy. The images of the specimen were collected with a Leica TCL SP2 confocal microscope system (Leica Microsystems, Germany).

\section{Reverse transcription-polymerase chain reaction (RT-PCR)}

Total cellular RNA was extracted using Trizol (Invitrogen). For RT-PCR, aliquots of $2 \mu \mathrm{g}$ RNA were subjected to cDNA synthesis with $200 \mathrm{U}$ of M-MLV reverse transcriptase and $0.5 \mu \mathrm{g}$ of oligo (dT) 15 primer (Promega, Madison, $\mathrm{WI})$. The cDNA in $2 \mu \mathrm{l}$ of the reaction mixture was amplified with $0.5 \mathrm{U}$ of GoTaq DNA polymerase (Promega, Madison, WI) and $10 \mathrm{pmol}$ each of sense and antisense primers as follows: TGF- $\beta$ type I receptor (190 bp) : sense 5'-GAACTTCCAACTACTGGTTT-3', antisense 5'-GATAAATCTCTGCCTCACGG-3', glyceraldehydes-3-phosphate dehydrogenase (GAPDH; 420 bp): sense 5'-TCCATGACAACTTTGGTATCG-3', antisense 5'-TGTAGCCAAATTCG TTGTCA-3'. The thermal cycle profile consisted of denaturation at $95^{\circ} \mathrm{C}$ for $30 \mathrm{~s}$, annealing at $52-55^{\circ} \mathrm{C}$ for $45 \mathrm{~s}$ depending on the primers used, and extension at $72^{\circ} \mathrm{C}$ for $45 \mathrm{~s}$. Each PCR reaction was carried out for 30 cycles, and PCR products were size fractionated on $1.2 \%$ ethidium bromide/agarose gel and photographed under UV transillumination.

\section{Transfection with small interfering RNA (siRNA)}

siRNA duplexes were synthesized, desalted, and purified by Samchully Pharm. (Siheung, GyeongGi, Korea) using Smad2 sense 5'-GUCCCAUGAAAAGACUUAATT-3' and antisense 5'-UUAAGUCUUUUCAUGGGACTT-3' primers. Nonspecific control siRNA (D-001206-13-05) was purchased from Dharmacon (Lafayette, CO). For siRNA experiments, hASCs were seeded on 6 well dishes at $70 \%$ confluence prior to transfection with siRNAs using Lipofectamine plus ${ }^{\mathrm{TM}}$ reagent (Invitrogen) according to the manufacturer's instructions. Briefly, Lipofectamine plus ${ }^{\mathrm{TM}}$ reagent was incubated with serum-free medium for $15 \mathrm{~min}$, and respective siRNAs were then added to the mixtures. After incubation for $15^{\mathrm{TM}} \mathrm{min}$ at room temperature, the 
mixtures were diluted with serum-free medium and added to each well. The final concentration of siRNAs in each well was $100 \mathrm{nM}$. After incubation of hASCs to serum-free medium containing siRNAs for $4 \mathrm{~h}$, the cells were cultured in growth medium for $24 \mathrm{~h}$, and the expression levels of Smad2 and GAPDH were then determined by western blot analysis.

\section{Lentiviral small hairpin RNA (shRNA) transduction}

pLKO.1-puro lentiviral vectors expressing TGF- $\beta$ type I receptor shRNA (TRCN0000039774) or nontarget control shRNA (SHC002) were purchased from Sigma-Aldrich. The functional sequence for TGF- $\beta$ type I receptor in the pLKO.1-puro shRNA lentiviral vector is CCGGGCGAGAACTATTGTGTTACAACTCGAGTTGTAACACAATAGT TCTCGCTTTTTG. To generate lentiviral particles, HEK293FT cells were co-transfected with the shRNA lentiviral plasmid (pLKO.1-puro) and ViraPower Lentiviral packaging mix (pLP1, pLP2, pLP-VSV-G; Invitrogen) using Lipofectamine plus ${ }^{\text {TM }}$ (Invitrogen) and the culture supernatants containing lentivirus were harvested at $48 \mathrm{~h}$ after transfection. For lentiviral transduction, hASCs were treated with the shRNA-expressing lentivirus in the presence of $5 \mu \mathrm{g} / \mathrm{ml}$ polybrene (Sigma-Aldrich) and stable cell lines expressing shRNA were generated by selection with puromycin $(10 \mu \mathrm{g} / \mathrm{ml})$. To ensure shRNA-mediated silencing of TGF- $\beta$ type I receptor expression, the mRNA levels of TGF- $\beta$ type I receptor and GAPDH were determined by RT-PCR.

\section{ELISA}

TGF- $\beta 1$ level was determined by a commercially available human TGF- $\beta 1$ ELISA kit (R\&D Systems) according to the manufacturer's instructions. In brief, $100 \mu \mathrm{l}$ of diluted capture antibody was added to each well of a 96-well microplate and then incubated overnight at room temperature for coating of the capture antibody. After blocking of each well with $100 \mu$ l of blocking solution, samples or standards were transferred to wells. After incubation for $2 \mathrm{~h}$ at room temperature, wells were washed with phosphate-buffered solution and $100 \mu \mathrm{l}$ of horseradish peroxidase-conjugated detection antibody was dispensed into each well. Following $2 \mathrm{~h}$ incubation at room temperature, the plate was washed, and then $100 \mu$ of substrate solution was added to each well. After incubation at room temperature for $20 \mathrm{~min}$, the reaction was stopped by the addition of $2 \mathrm{~N} \mathrm{H}_{2} \mathrm{SO}_{4}$ to each well and the absorbance of each solution at $450 \mathrm{~nm}$ was determined by using a PowerWavex microplate spectrophotometer (Bio-Tek Instruments, Winooski, VT). The concentration of TGF- $\beta 1$ in the samples is determined by comparison to the standard curve.

\section{Western blot analysis}

hASCs were treated under appropriate conditions, washed with PBS, and then lysed in lysis buffer $(20 \mathrm{mM}$ Tris-HCL, $1 \mathrm{mM}$ EGTA, $1 \mathrm{mM}$ EDTA, $10 \mathrm{mM} \mathrm{NaCl}, 0.1 \mathrm{mM}$ phenylmethylsulfonyl fluoride, $1 \mathrm{mM} \mathrm{Na} \mathrm{VO}_{4}, 30 \mathrm{mM}$ sodium pyrophosphate, $25 \mathrm{mM} \beta$-glycerophosphate, $1 \%$ Triton $X-100, \mathrm{pH} 7.4$ ). The lysates were resolved by $10 \%$ sodium dodecyl sulfate-polyacrylamide gel electrophoresis (SDS-PAGE). Separated proteins were transferred onto a nitrocellulose membrane and stained with $0.1 \%$ Ponceau $S$ solution (Sigma-Aldrich). After blocking with $5 \%$ nonfat milk, the membranes were immunoblotted with primary antibodies, and the bound antibodies were visualized with horseradish peroxidase-conjugated secondary antibodies using the enhanced chemiluminescence Western blotting system (Amersham Biosciences, Piscataway, NJ).

\section{Depletion of TGF- $\beta 1$ from RA-SF with anti-TGF- $\beta 1$ antibody}

TGF- $\beta 1$ was immunodepleted from RA-SF by immunoprecipitation with anti-TGF- $\beta 1$ antibody. In brief, aliquots (30 $\mu \mathrm{l})$ of a suspension ( $50 \%$ slurry) of protein G-agarose beads (Sigma-Aldrich) in PBS were mixed with $0.2 \mu \mathrm{g}$ of anti-TGF- $\beta 1$ or normal mouse antibodies at $4^{\circ} \mathrm{C}$ for $1 \mathrm{~h}$ with intermittent shaking. After recovery by centrifugation, the beads were washed three times and used for immunodepletion of TGF-B1 from RA-SF. 1\% RA-SF or 0.2 $\mathrm{ng} / \mathrm{ml}$ TGF- $\beta 1$ were incubated with the protein $\mathrm{G}$-agarose beads immobilized with anti-TGF- $\beta 1$ or normal mouse antibodies for $1 \mathrm{~h}$ at $4^{\circ} \mathrm{C}$. The immune complexes absorbed to protein $\mathrm{G}$-agarose beads were precipitated by centrifugation. The resultant supernatants were collected and immediately used for experiments.

\section{Adenoviral infection}

Recombinant adenoviruses expressing Smad7 or LacZ were kindly provided by Dr. Kohei Miyazono (Tokyo University, Japan) and were used individually at a multiplicity of infection of 50 as described previously (Fujii et al., 1999).

\section{Statistical analysis}

The results of multiple observations are presented as mean $\pm S D$. For multivariate data analysis, group differences were assessed with two-way ANOVA, followed by post hoc comparisons tested with Scheffe's method.

\section{Acknowledgements}

This work was supported by the Bio Research Grant funded by the Pusan National University (PNU, Bio Research Fund; PNU- 2008-059-7000).

\section{References}

Attisano L, Wrana JL. Smads as transcriptional co-modulators. Curr Opin Cell Biol 2000;12:235-43

Barry FP, Murphy JM. Mesenchymal stem cells: clinical applications and biological characterization. Int J Biochem Cell Biol 2004;36:568-84 
Bouffi C, Djouad F, Mathieu M, Noel D, Jorgensen C. Multipotent mesenchymal stromal cells and rheumatoid arthritis: risk or benefit? Rheumatology (Oxford) 2009; 48:1185-9

Buckley CD. Michael Mason prize essay 2003. Why do leucocytes accumulate within chronically inflamed joints? Rheumatology (Oxford) 2003;42:1433-44

de Caestecker $M$. The transforming growth factor-beta superfamily of receptors. Cytokine Growth Factor Rev 2004; 15:1-11

De Wever O, Demetter P, Mareel M, Bracke M. Stromal myofibroblasts are drivers of invasive cancer growth. Int J Cancer 2008;123:2229-38

Djouad F, Bouffi C, Ghannam S, Noel D, Jorgensen C. Mesenchymal stem cells: innovative therapeutic tools for rheumatic diseases. Nat Rev Rheumatol 2009;5:392-9

Feldmann M, Brennan FM, Maini RN. Rheumatoid arthritis Cell 1996;85:307-10

Fuji M, Takeda K, Imamura T, Aoki H, Sampath TK, Enomoto S, Kawabata M, Kato M, Ichijo H, Miyazono K. Roles of bone morphogenetic protein type I receptors and Smad proteins in osteoblast and chondroblast differentiation. Mol Biol Cell 1999;10:3801-13

Gabbiani G. The myofibroblast in wound healing and fibrocontractive diseases. J Pathol 2003;200:500-3

Ghosh P, Guidolin D. Potential mechanism of action of intra-articular hyaluronan therapy in osteoarthritis: are the effects molecular weight dependent? Semin Arthritis Rheum 2002;32:10-37

Jeon ES, Moon HJ, Lee MJ, Song HY, Kim YM, Bae YC, Jung JS, Kim JH. Sphingosylphosphorylcholine induces differentiation of human mesenchymal stem cells into smoothmuscle-like cells through a TGF-beta-dependent mechanism. J Cell Sci 2006;119:4994-5005

Jeon ES, Moon HJ, Lee MJ, Song HY, Kim YM, Cho M, Suh DS, Yoon MS, Chang CL, Jung JS, Kim JH. Cancer-derived lysophosphatidic acid stimulates differentiation of human mesenchymal stem cells to myofibroblast-like cells. Stem Cells 2008;26:789-97

Jones EA, English A, Henshaw K, Kinsey SE, Markham AF, Emery P, McGonagle D. Enumeration and phenotypic characterization of synovial fluid multipotential mesenchymal progenitor cells in inflammatory and degenerative arthritis. Arthritis Rheum 2004;50:817-27

Kasperkovitz PV, Timmer TC, Smeets TJ, Verbeet NL, Tak $P P$, van Baarsen LG, Baltus B, Huizinga TW, Pieterman E, Fero M, Firestein GS, van der Pouw Kraan TC, Verweij CL. Fibroblast-like synoviocytes derived from patients with rheumatoid arthritis show the imprint of synovial tissue heterogeneity: evidence of a link between an increased myofibroblast-like phenotype and high-inflammation synovitis. Arthritis Rheum 2005;52:430-41

Kastrinaki MC, Papadaki HA. Mesenchymal stromal cells in rheumatoid arthritis: biological properties and clinical applications. Curr Stem Cell Res Ther 2009;4:61-9

Marinova-Mutafchieva L, Williams RO, Funa K, Maini RN, Zvaifler NJ. Inflammation is preceded by tumor necrosis factor-dependent infiltration of mesenchymal cells in experimental arthritis. Arthritis Rheum 2002;46:507-13

Mattey DL, Dawes PT, Nixon NB, Slater H. Transforming growth factor beta 1 and interleukin 4 induced alpha smooth muscle actin expression and myofibroblast-like differentiation in human synovial fibroblasts in vitro: modulation by basic fibroblast growth factor. Ann Rheum Dis 1997;56: 426-31

Pittenger MF, Mackay AM, Beck SC, Jaiswal RK, Douglas R, Mosca JD, Moorman MA, Simonetti DW, Craig S, Marshak DR. Multilineage potential of adult human mesenchymal stem cells. Science 1999;284:143-7

Pohlers D, Beyer A, Koczan D, Wilhelm T, Thiesen HJ, Kinne RW. Constitutive upregulation of the transforming growth factor-beta pathway in rheumatoid arthritis synovial fibroblasts. Arthritis Res Ther 2007;9:R59

Pohlers D, Brenmoehl J, Loffler I, Muller CK, Leipner C, Schultze-Mosgau S, Stallmach A, Kinne RW, Wolf G. TGF-beta and fibrosis in different organs - molecular pathway imprints. Biochim Biophys Acta 2009;1792:746-56

Prockop DJ. Marrow stromal cells as stem cells for nonhematopoietic tissues. Science 1997;276:71-4

Salvador G, Sanmarti R, Gil-Torregrosa B, Garcia-Peiro A, Rodriguez-Cros JR, Canete JD. Synovial vascular patterns and angiogenic factors expression in synovial tissue and serum of patients with rheumatoid arthritis. Rheumatology (Oxford) 2006;45:966-71

Scrivo R, Di Franco M, Spadaro A, Valesini G. The immunology of rheumatoid arthritis. Ann N Y Acad Sci 2007; 1108:312-22

Shi Y, Massague J. Mechanisms of TGF-beta signaling from cell membrane to the nucleus. Cell 2003;113:685-700

Short B, Brouard N, Occhiodoro-Scott T, Ramakrishnan A, Simmons PJ. Mesenchymal stem cells. Arch Med Res 2003; 34:565-71

Song HY, Lee MJ, Kim MY, Kim KH, Lee IH, Shin SH, Lee JS, Kim JH. Lysophosphatidic acid mediates migration of human mesenchymal stem cells stimulated by synovial fluid of patients with rheumatoid arthritis. Biochim Biophys Acta 2010;1801:23-30

Steenvoorden MM, Tolboom TC, van der PG, Lowik C, Visser CP, DeGroot J, Gittenberger-DeGroot AC, DeRuiter MC, Wisse BJ, Huizinga TW, Toes RE. Transition of healthy to diseased synovial tissue in rheumatoid arthritis is associated with gain of mesenchymal/fibrotic characteristics. Arthritis Res Ther 2006; 8:R165

Szekanecz Z, Strieter RM, Kunkel SL, Koch AE. Chemokines in rheumatoid arthritis. Springer Semin Immunopathol 1998; 20:115-32

Tanasescu C, Jurcut C, Jurcut R, Ginghina C. Vascular disease in rheumatoid arthritis: from subclinical lesions to cardiovascular risk. Eur J Intern Med 2009;20:348-54

ten Dijke P, Hill CS. New insights into TGF-beta-Smad signalling. Trends Biochem Sci 2004;29:265-73

Wahl SM. Transforming growth factor-beta: innately bipolar. Curr Opin Immunol 2007;19:55-62 